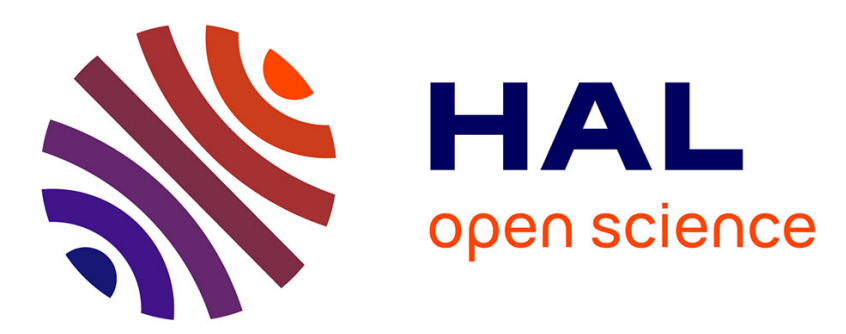

\title{
Impact of long separating distances on the energy production of two interacting wave energy converters
}

A. Babarit

\section{To cite this version:}

A. Babarit. Impact of long separating distances on the energy production of two interacting wave energy converters. Ocean Engineering, 2010, 37, pp.718-729. 10.1016/j.oceaneng.2010.02.002 . hal01145086

\section{HAL Id: hal-01145086 \\ https://hal.science/hal-01145086}

Submitted on 9 Jun 2019

HAL is a multi-disciplinary open access archive for the deposit and dissemination of scientific research documents, whether they are published or not. The documents may come from teaching and research institutions in France or abroad, or from public or private research centers.
L'archive ouverte pluridisciplinaire HAL, est destinée au dépôt et à la diffusion de documents scientifiques de niveau recherche, publiés ou non, émanant des établissements d'enseignement et de recherche français ou étrangers, des laboratoires publics ou privés. 


\title{
Impact of long separating distances on the energy production of two interacting wave energy converters
}

\author{
Aurélien Babarit \\ Laboratoire de Mécanique des Fluides (CNRS UMR6598), Ecole Centrale de Nantes, 1 Rue de la Noë B.P 92101, 44321 Nantes Cedex 3, France
}

\begin{abstract}
In this paper, wave farms composed of two either surging or heaving wave energy converters are considered. Using a numerical model which takes into account wave interactions, the impact on the absorbed wave power of the separating distance between the two systems and the wave direction is studied. In regular waves, a modified $q_{m o d}$ factor is introduced and it is found to be more relevant than the usual $q$ factor for identifying this impact. Then, it is shown that, asymptotically, the alteration of the energy absorption due to wave interaction effects decreases with the square root of the distance. This is a slow decay, which leads to a still significant modification of the wave energy absorption at long distance (up to $15 \%$ at a distance of $2000 \mathrm{~m}$ ). In irregular waves, it is shown that constructive and destructive effects compensate each other, particularly when considering the mean annual power. It leads to a smaller impact of the wave interactions on the absorbed energy and shorter distances (smaller than $10 \%$ for distances greater than $400 \mathrm{~m}$ ). Finally, conclusions on if wave interactions should be taken into account or not when designing a wave farm are drawn in function of the distance.
\end{abstract}

\section{Introduction}

Wave energy converters (WECs) are designed to be deployed in large arrays composed of many systems. In such arrays, each single system interacts with all the others by absorbing, radiating and diffracting waves. These wave interactions have an impact on the energy output from arrays, which motivated many research studies over the last decades.

The effect of array interactions on the energy production is usually quantified by the $q$ factor, defined as the ratio between the output power from an array of $N$ systems divided by $N$ times the output power from a single isolated system. If $q<1$, it means that the averaged energy production of each system in the array is lower than the energy production of isolated systems. Hence, the wave interferences have a destructive effect on the output power of the wave farm. Reversely, if $q>1$, the effect is constructive.

In the pioneering work of Budal (1977), Evans (1979) and Falnes (1980), it has been shown that the $q$ factor can be either higher or lower than 1 depending on the wave frequencies and the array layout. This means that it can exist farm arrangements in which the energy production from a sum of WECs is more than the sum of the energy production of each single WEC. However, in Thomas and Evans (1981), it is stated that farm layouts should be designed in order to minimise destructive interferences for practical applications. Since then, many studies have been

E-mail address: aurelien.babarit@ec-nantes.fr conducted by various authors on linear arrays of small devices (Simon, 1982; Mclver and Evans, 1984; Mavrakos and Mclver, 1997; Falcão, 2002; Justino and Clément, 2003; Child and Venugopal, 2007; Cruz et al., 2009; Weller et al., 2009).

In most of these studies, only closely spaced arrays are considered. In such arrays, wave interactions are strong because each WEC feels the wave perturbation coming from one or several others WECs in the array. It is well known (Falnes, 2002) that the wave perturbation is composed of a near field part which decays with the inverse distance to the body which generated it; and a far field part which decays with the squareroot of that distance. Hence, when the distance between the WECs in the array is sufficiently large, wave interactions become negligible.

For practical reasons (moorings for example), arrays of WECs can become sparse, with typical separating distances of a few hundred meters. Taking into account the considerations of previous paragraph, one could ask if the WECs are far enough in order to neglect the wave interactions. This is the question addressed in this study by considering two arrays of two generic wave energy converters-one heaving and one surging devicelocated at several different distances one from the other.

In the first part of this paper, a numerical model of the array is derived in the frequency domain. In the second part, results of numerical simulation are presented, both in regular and irregular waves. In conclusion, range of distances for which it seems to be worth taking into account wave interactions or not are proposed. 


\section{Methods}

\subsection{Equation of motion of two wave energy converters}

Let us consider two basic arrays of wave energy converters, Fig. 1. The first array, array I, is composed of two semi-submerged cylinders and the second array, array II, of two semi-submerged rectangular shaped floating bodies. The diameter of each cylinder is taken equal to $10 \mathrm{~m}$ and their draught is equal to $10 \mathrm{~m}$, corresponding to a displacement $V_{1}$ of around $785 \mathrm{~m}^{3}$. It is assumed that both cylinders can move only in the heave motion $z$ (i.e. along the vertical axis), with all other degrees of freedom ideally restricted. For the second array, the width and draught of the two bodies are taken equal to $10 \mathrm{~m}$ in order to have the same surface facing the waves and the length is taken equal to $7.85 \mathrm{~m}$ in order to have a volume similar as the one of the cylinders. Their motion is restricted to the surge motion $x$, all other degrees of freedom being ideally restricted. For both arrays, an idealised power take off (PTO) is considered, composed of a linear spring and damper system with stiffness $k_{\text {Рто }}$ and damping coefficient $b_{\text {РТО. }}$

Let us note with index 1 and 2 all quantities related, respectively, to the first and with the second system in each array. Let $z_{1}$ and $z_{2}$ be the heave motion of each buoy in the first array, and $x_{1}$ and $x_{2}$ be the surge motions in the second one. Let $\mathbf{X}=\left(z_{1} z_{2}\right)^{t}$ (respectively $\left.\left(x_{1} x_{2}\right)^{t}\right)$ be the position vector of the whole array. Assuming the fluid to be non-viscid and incompressible, the flow to be irrotational, and the amplitude of motions and waves to be sufficiently small in comparison with the wavelength and the dimensions of the bodies, the classical linearised potential theory can be used as a framework for calculation of the fluid-structure interactions. Hence, one can write the equation of motion of the WEC in the frequency domain for unitary wave amplitude and a wave frequency $\omega$ :

$(\mathbf{M}+\mathbf{A M}(\omega)) \ddot{\mathbf{X}}+\left(\mathbf{B}_{\text {PтO }}+\mathbf{B}(\omega)\right) \dot{\mathbf{X}}+\left(\mathbf{K}_{H}+\mathbf{K}_{A}+\mathbf{K}_{\text {PTO }}\right) \mathbf{X}=\mathbf{F}_{e x}$

with:

- $\mathbf{X}=\mathfrak{R}\left(\bar{X} e^{i \omega t}\right)$ and $\dot{\mathbf{X}}, \ddot{\mathbf{X}}$ being, respectively, the velocity and acceleration vectors of the WECs.

- $\mathbf{M}=\left(\begin{array}{cc}m_{1} & 0 \\ 0 & m_{2}\end{array}\right)$ the mass matrix of the system. As it is considered identical bodies in each of both arrays, $m_{1}=m_{2}=785 \mathrm{t}$.
- $\mathbf{K}_{H}=\left(\begin{array}{cc}k h_{1} & 0 \\ 0 & k h_{2}\end{array}\right)$ the hydrostatic stiffness matrix of the system. In the array composed of heaving cylinders, $k h_{1}=k h_{2}=770 \mathrm{kN}$ $\mathrm{m}^{-1}$. In the array composed of surging barges, $k h_{1}=k h_{2}=0 \mathrm{kN}$ $\mathrm{m}^{-1}$.

- $\mathbf{K}_{A}$ an additional stiffness matrix which represents the action of possible moorings. In this study, it was neglected, i.e. $\mathbf{K}_{A}=\mathbf{0}$ in both arrays.

- $\mathbf{A M}(\omega)=\left(\begin{array}{ll}a m_{11} & a m_{12} \\ a m_{21} & a m_{22}\end{array}\right)$ the added mass matrix and $\mathbf{B}(\omega)=\left(\begin{array}{ll}b_{11} & b_{12} \\ b_{21} & b_{22}\end{array}\right)$ the wave damping matrix which represent the radiation of waves by the body when it moves. In these matrices, the nondiagonal terms are not anymore equal to 0 . They represent the pressure force measured on one body due to the radiated wave associated with a motion of the other one. For obvious symmetry reasons, $a m_{11}=a m_{22}, a m_{12}=a m_{21}, b_{11}=b_{22}, b_{12}=b_{21}$.

- $\mathbf{F}_{e x}=\mathfrak{R}\left(\bar{F}_{e x} e^{i \omega t}\right)$ is the excitation vector per unit of wave amplitude, associated to the action of incident and diffracted wave fields upon the WECs.

- $\mathbf{K}_{\text {PTO }}=\left(\begin{array}{cc}k_{\text {PTO }} & 0 \\ 0 & k_{\text {PTO }}\end{array}\right)$ and $\mathbf{B}_{\text {PTO }}=\left(\begin{array}{cc}b_{\text {PTO }} & 0 \\ 0 & b_{\text {PTO }}\end{array}\right)$ are the matrices associated with the action of the PTOs. In array I, $k_{\text {PTO }}$ is set equal to 0 . In array II, $k_{\text {Рто }}$ is tuned in order the surging barges to have the same natural frequency than the heaving cylinders of array

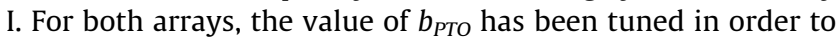
achieve the maximum energy absorption at the natural frequency $\omega_{0}$ of an isolated device. Following Falnes (2002), it has been set equal to the wave damping coefficient, i.e: $b_{\text {PTO }}=b_{\text {isolated }}\left(\omega_{0}\right)$.

In regular waves, the mean power extracted by each buoy in the array per unit of wave amplitude is given by

$p_{i}=\frac{1}{2} b_{P T O} \omega^{2}\left|\overline{X_{i}}\right|^{2}$

with $i \in 1,2$. For the whole array, the mean absorbed power is

$p=\sum_{i=1}^{2} p_{i}$

In irregular waves, characterised by a wave energy spectrum $S$, the mean power extracted by each buoy is given by

$P_{i}=\int_{0}^{+\infty} S(\omega) p_{i}(\omega) d \omega$
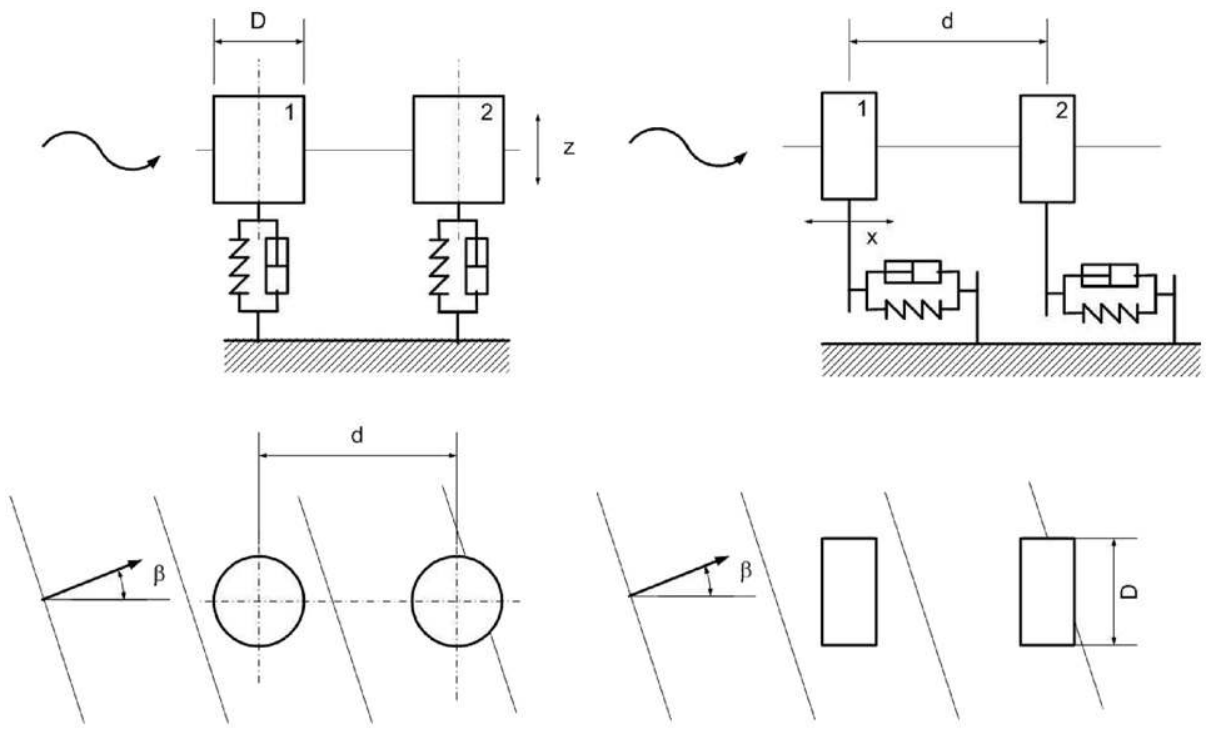

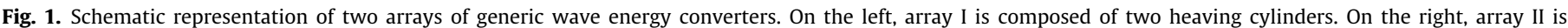
composed of two surging barges. 
In this study, the standard Jonswap energy spectrum $S\left(H_{S}, T_{1}, \gamma\right)$ (Molin, 2002) has been used, in which $H_{S}$ is the significant wave, $T_{1}$ is the peak period and $\gamma$ gives the frequency spreading. The frequency spreading parameter has been taken equal to $\gamma=3.3$ in all sea states $\left(H_{S}, T_{1}\right)$.

Using wave data statistics at a given location, one can calculate the annual mean wave power absorbed by each body $\left\langle P_{i}\right\rangle$. Let $C\left(H_{S}, T_{1}\right)$ be the probability of occurrence of each sea state $\left(H_{S}, T_{1}\right)$. $\left\langle P_{i}\right\rangle$ is given by

$\left\langle P_{i}\right\rangle=\sum_{\left(H_{S}, T_{1}\right)} C\left(H_{S}, T_{1}\right) P_{i}\left(H_{S}, T_{1}\right)$

For the whole array, the mean absorbed power is

$\langle P\rangle=\sum_{i=1}^{2}\left\langle P_{i}\right\rangle$

In this study, wave data statistics measured by a datawell a few kilometers offshore from Yeu island (on the French Atlantic coast) were used, see Fig. 2.

\subsection{Numerical model}

A numerical model was written in Fortran to solve Eq. (1). The hydrodynamic coefficients $\mathbf{A M}(\omega), \mathbf{B}(\omega)$ and $\overline{\mathbf{F}}_{e x}(\omega)$ were calculated using the BEM based code AQUADYN (Delhommeau, 1993). In order to minimise the number of parameters, all calculations were performed in deep water. Although the water depth may have an influence on the results, it has been chosen not to consider this parameter in this study to keep it simple.

The hydrodynamic coefficients calculated using AQUADYN are plotted in Fig. 3 in the case of array I, the WECs being separated by a distance of 10 diameters. For the sake of comparison, the hydrodynamic coefficients of a single isolated body have also been plotted. One could notice that the amplitude of the crossed coupled wave damping coefficients $b_{12}$ can be of an order of magnitude close to $b_{11}$, which shows the interaction between the buoys in the velocity-proportional part of the radiation force. This interaction is not as strong in the acceleration part of the radiation force, in which the crossed coupled added mass coefficients are, at most, 10 times smaller than the diagonal ones.

About the wave excitation force coefficient, the interaction effect appears to be small. One could have expected that the main effect of wave interactions would have been experienced by the second system which lies in the wake of the first system (which meets the wave first) but it appears that it happens the contrary. One can see that the wave excitation force coefficient of the

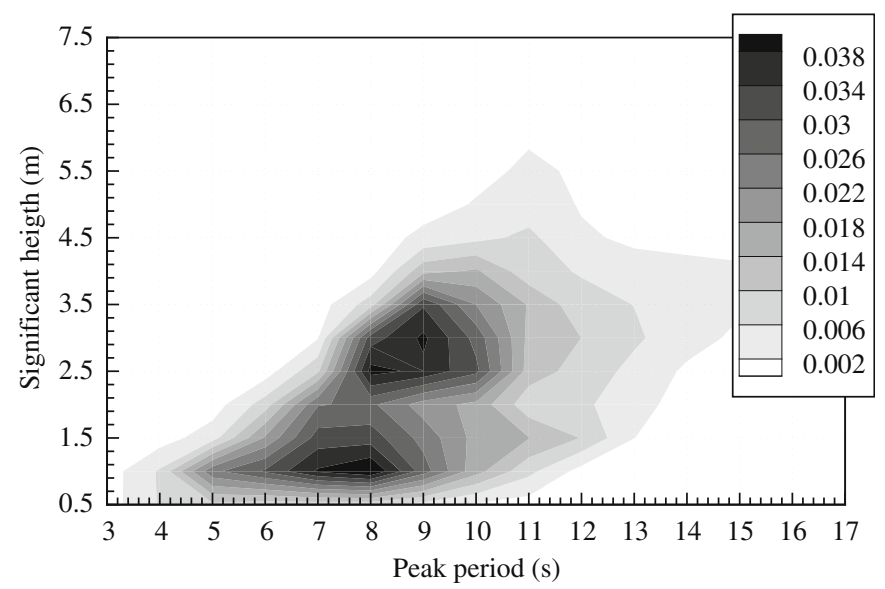

Fig. 2. Wave scatter diagram for Yeu island $\left(46^{\circ} 41.45^{\prime} \mathrm{N}, 2^{\circ} 25.65^{\prime} \mathrm{W}\right)$.
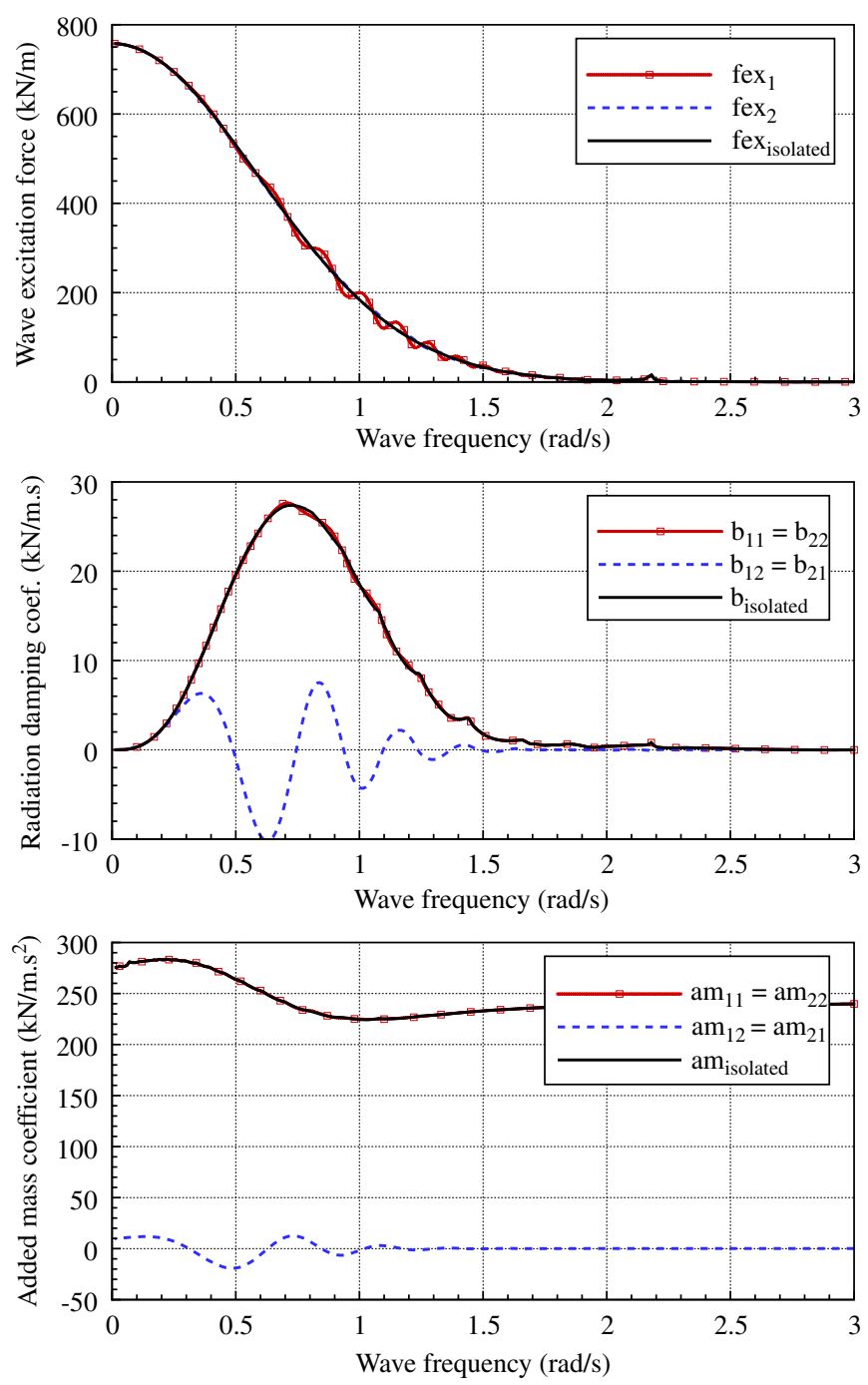

Fig. 3. Comparisons of the amplitude of the wave excitation force coefficients (top), wave damping coefficients (middle) and added mass coefficients (bottom) in array I with the ones of a single isolated heaving cylinder. Wave direction is $0^{\circ}$. Separating distance between the two bodies is 10 diameters, i.e. $100 \mathrm{~m}$.

second body is not disturbed in comparison with the one of a single isolated system, whereas it exists a small wavy difference for the first system in the range of frequencies $[0.5,1.5] \mathrm{rad} \mathrm{s}^{-1}$. This is a quite surprising result which was already observed by Child and Venugopal (2007) in the same case of two interacting cylinders.

In Fig. 4, the same hydrodynamic coefficients are plotted with the same distance between the WECs (i.e. $100 \mathrm{~m}$ ) but in the case of array II. One can see that the results for these surging devices are very different from the previous ones. Now, the wave interactions appear to be much stronger both on the wave damping and added mass crossed coefficients, and on the wave excitation force. Again, the effect on this last coefficient appears to be stronger on the first body than on the second one.

Fig. 5 shows RAOs and capture width ratios $w$ of both heaving and surging WECs, $w$ being defined as the ratio of the power absorbed by the system divided by the incident power in the width of each system. The natural period of the surging device matches with the one of the heaving WEC, which has been obtained by tuning the PTO stiffness $k_{\text {PTO }}$ of the surging device to $10^{4} \mathrm{kN} \mathrm{m}^{-1}$. Table 1 gives the other PTO parameters which have been taken in these calculations. 

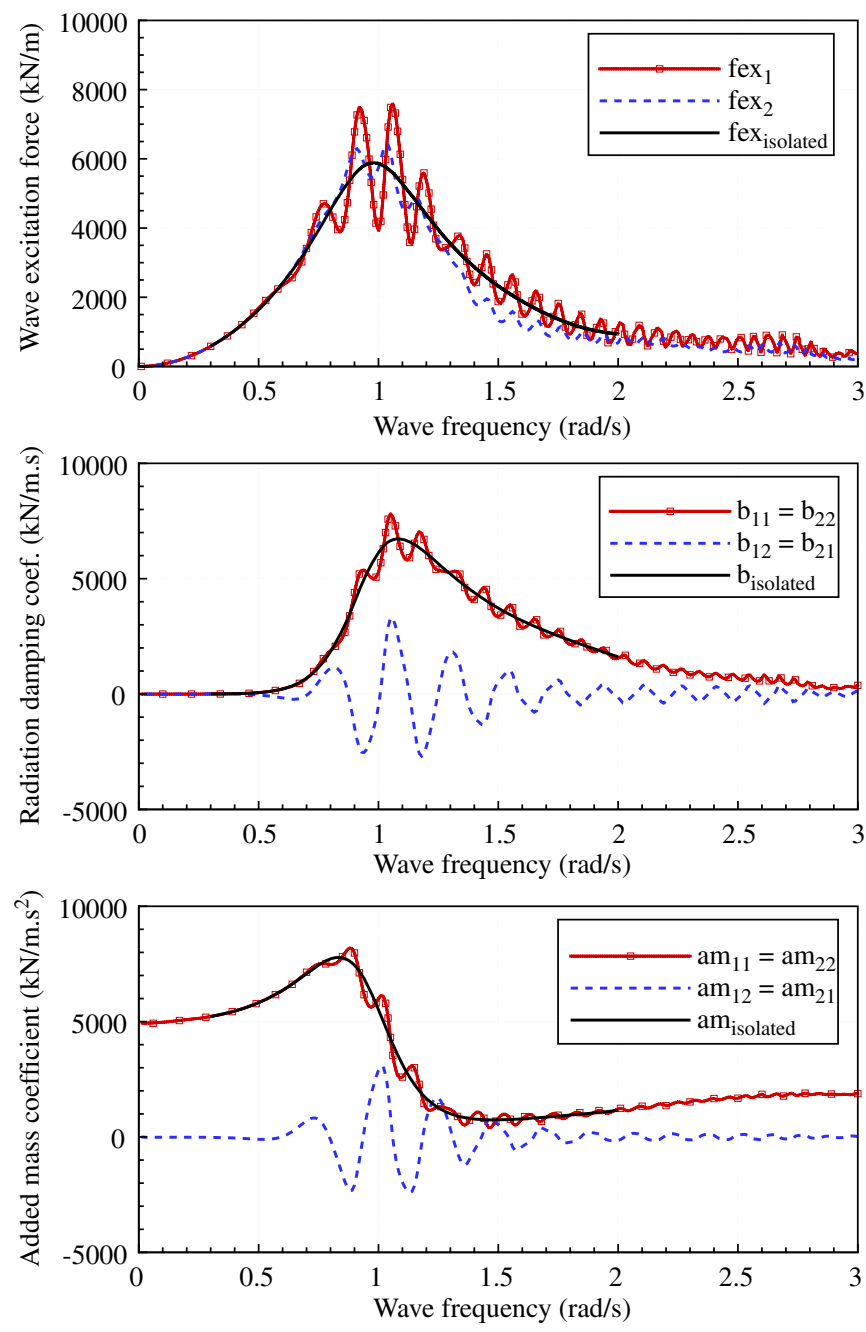

Fig. 4. Comparisons of the amplitude of the wave excitation force coefficients (top), wave damping coefficients (middle) and added mass coefficients (bottom) in array II with the ones of a single isolated surging device. Wave direction is 0 . Separating distance between the two bodies is 10 times the width of the system, i.e. $100 \mathrm{~m}$.

One can see in this figure that the capture width ratio of the surging WEC is much higher than the one of the heaving device, whereas the amplitude of the motion is much lower. At resonance, one could notice that it is around twice the capture width of the heaving device, which was expected since the theoretical maximum capture width of a point absorber in surge is double the one in heave (Falnes, 2002).

Reminding that the total displacements of both systems are the same, this last figure suggests that a surging device would be more efficient for wave energy conversion than a heaving one, at least for the dimensions considered. However, these considerations are out of the scope of this study and they have not been investigated deeper here.

\subsection{Layout of the array}

In the layouts shown in Fig. 1 for the array, there are two parameters which could affect the mean absorbed power by the array $P$. They are the distance $d$ between the two systems and the main direction of propagation of the incident waves $\beta$. In this study, both are considered.
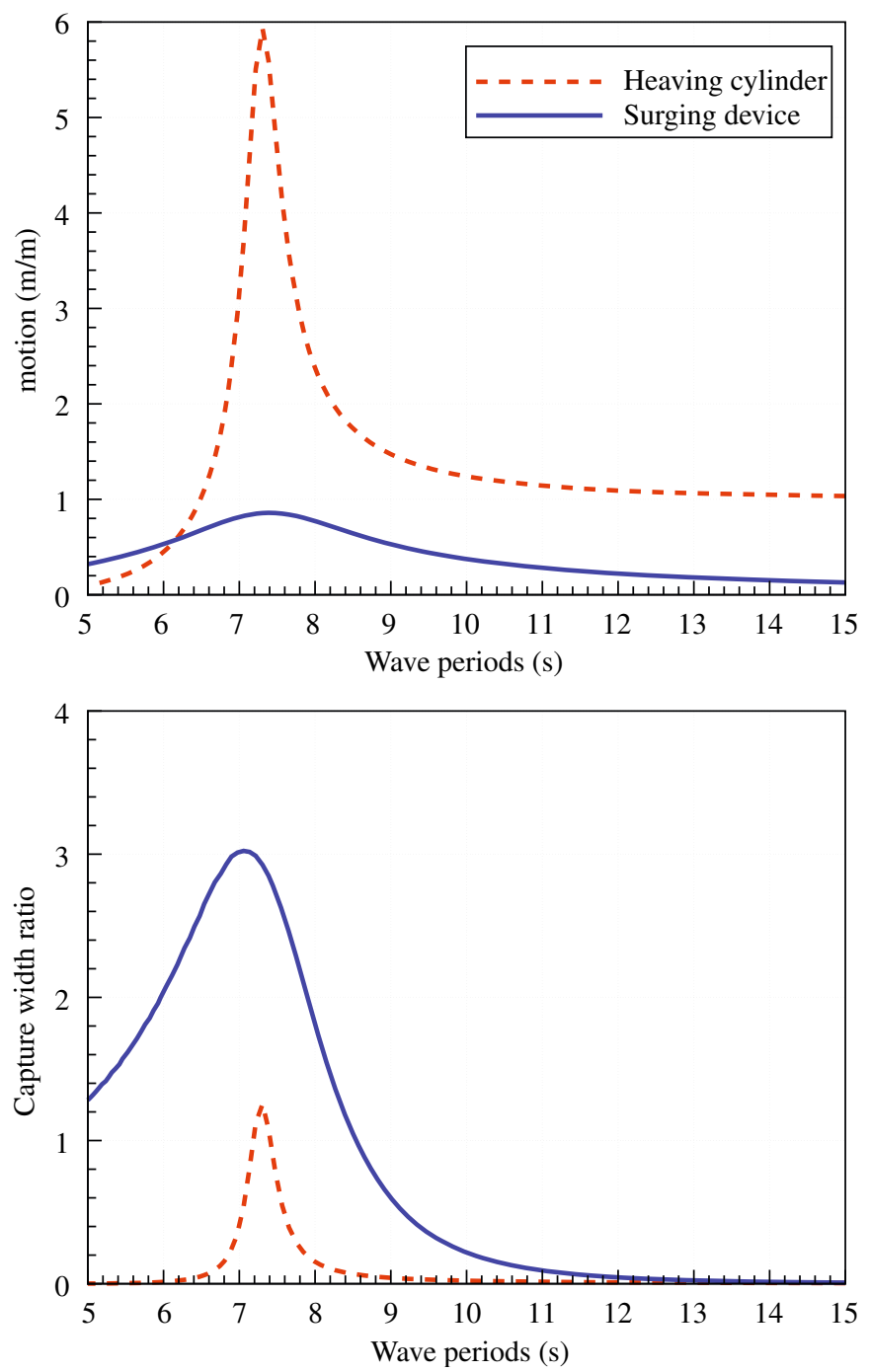

Fig. 5. RAOs (top) and capture width ratio (bottom) of both considered WECs, isolated. Wave direction is $0^{\circ}$.

Table 1

PTO parameters.

\begin{tabular}{llll}
\hline & $k_{\text {РTO }}\left(\mathrm{kN} \mathrm{m}^{-1}\right)$ & $b_{\text {PTO }}\left(\mathrm{kN} \mathrm{s} \mathrm{m}^{-1}\right)$ & Natural period $T_{0}(\mathrm{~s})$ \\
\hline Surging WEC & $10^{4}$ & $3.026 \times 10^{3}$ & 7.35 \\
Heaving WEC & 0 & 27 & 7.29 \\
\hline
\end{tabular}

\section{Results}

\subsection{Regular waves}

\subsubsection{Modified $q_{\text {mod }}$ factor}

In Fig. 6, the capture width ratio and standard $q$ factors for both bodies 1 and 2 of array I have been plotted in regular waves. The wave direction is $0^{\circ}$ and the distance between the two systems is taken equal to $100 \mathrm{~m}$. For the sake of comparison, the capture width ratio of a single isolated system has also been plotted in this figure.

Let us consider first only the $q$ factors. One could say that wave interactions are significant for all the wave periods below $8 \mathrm{~s}$ and that, again, the wave interaction effect is stronger on the system 

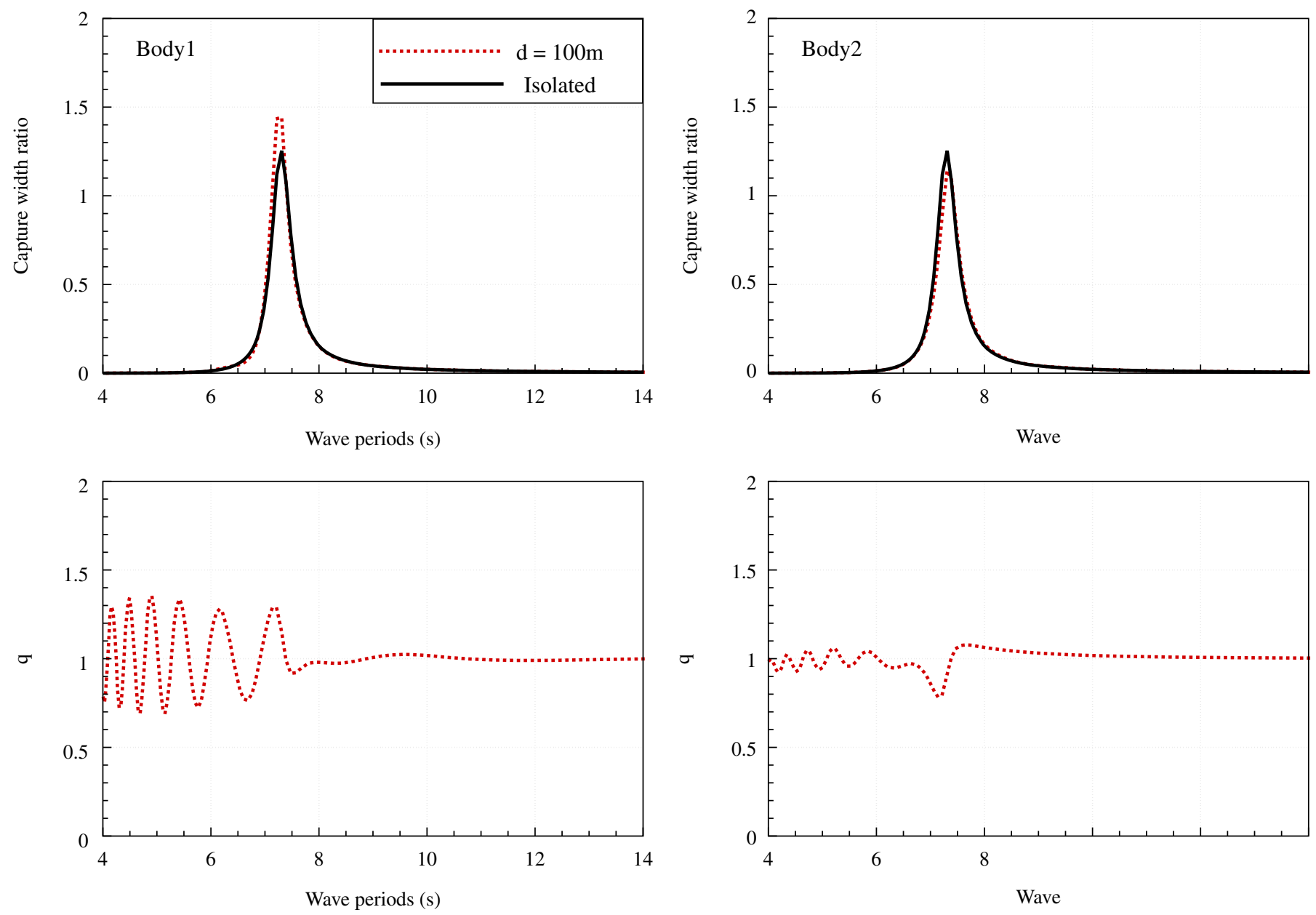

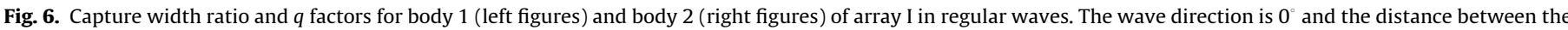
two systems is taken equal to $100 \mathrm{~m}$. For the sake of comparison, the capture width ratio of a single isolated system has been plotted too.

which meets the wave first than on the second one. However, considering now the two graphs showing the capture width ratio of each body, one can see that, if the second statement is true, the first one is kind of specious, since the capture width from the wave is very low as soon as the wave period is off-resonance. Actually, on these capture width ratio graphs, one could conclude that the wave interaction effect appears to be noticeable only when the systems are close and only when the wave period is close to the natural period of the system, for which the absorbed power is significant.

This shows that the $q$ factor is not sufficient when considering wave interactions in an array of WECs because it hides the real amount of absorbed power. Hence, one could use a modified factor $q_{\text {mod }}$ instead of the usual $q$ factor, defined as the ratio of the difference between the power absorbed $P_{i}$ by the system $\mathrm{i}$ in the array minus the power absorbed by an isolated system $P_{\text {isolated, }}$ divided by the maximum absorbed power by an isolated system $\max _{T} P_{\text {isolated }}(T)$ :

$q_{\text {mod }}=\frac{P_{i}-P_{\text {isolated }}}{\max _{T} P_{\text {isolated }}(T)}$

If $q_{\text {mod }}$ is positive, it means that the wave interaction has a constructive effect on the energy production. Reversely, if $q_{\text {mod }}$ is negative, the effect is destructive.

Fig. 7 shows this $q_{\text {mod }}$ factor for the previous array. One can see that the periods for which the absorbed power is very low are now filtered out. One would draw the same conclusions from these graphs as the ones which were made by considering the capture width ratios on Fig. 6.

Fig. 8 shows capture width ratios and $q_{\text {mod }}$ factors for body 1 (left figures) and body 2 (right figures) in regular waves, but now in the case of array II. Again, both bodies are separated with a distance of $100 \mathrm{~m}$ and the wave direction is $0^{\circ}$.

From the effect of wave interactions on the absorbed power point of view, the same conclusions can be drawn from both capture width ratios and $q_{\text {mod }}$ factors:

- The wave interactions effect is much more important than in the case of array I and it exists on the whole bandwidth of the system.

- The wave interactions effect is oscillating for body 1 . It can be constructive (up to $70 \%$ at natural period) or destructive (down to $55 \%$ ) depending on the wave period. For body 2 , it is destructive for almost all wave periods, with a minimum at $50 \%$.

Hence, from these two examples, one can see that the modified version of the $q$ factor seems to be a better parameter for characterising the impact of wave interactions on absorbed power than the usual definition of the $q$ factor.

\subsubsection{Influence of the distance}

Let us consider array I with long distances between the two systems, from 2 to $20 \mathrm{~km}$ between each body. For each distance, 

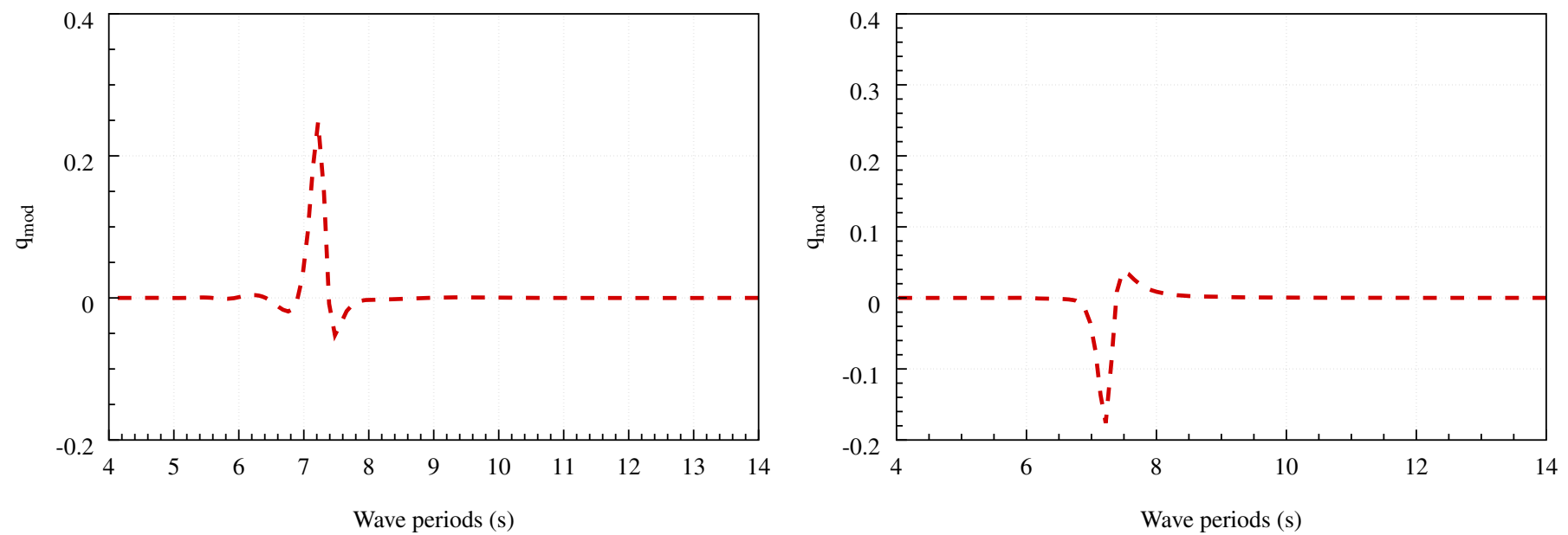

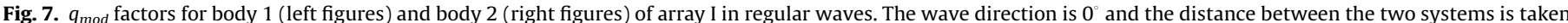
equal to $100 \mathrm{~m}$.
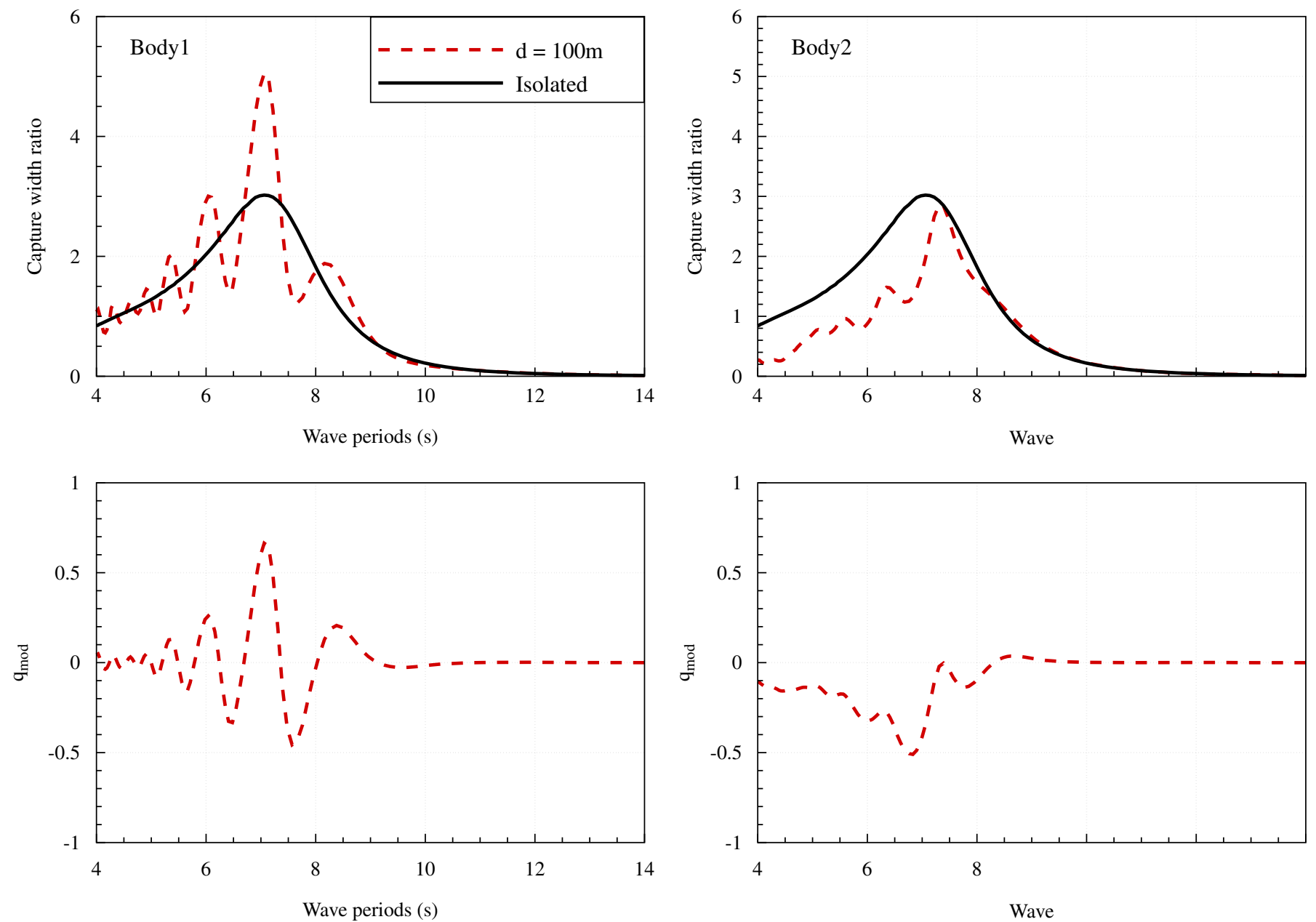

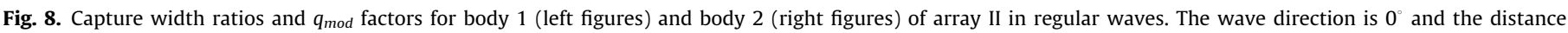
between the two systems is taken equal to $100 \mathrm{~m}$.

the $q_{\text {mod }}$ factors of each body in each array have been computed for wave periods between 3 and 20 s. To get a synthetic view of the wave interactions effect on the absorbed power in function of the distance, only the maximum and minimum of these $q_{\text {mod }}$ factors for each body have been plotted in Fig. 9.
One can see that, for both bodies, wave interaction effect can be positive ( $\max q_{\text {mod }}>0$ ) or negative $\left(\min q_{\text {mod }}<0\right.$ ), depending on the wave period. As it was expected, the interaction decreases regularly with the distance separating the two bodies. Best fit for these curves was found to be a polynomial law with power 

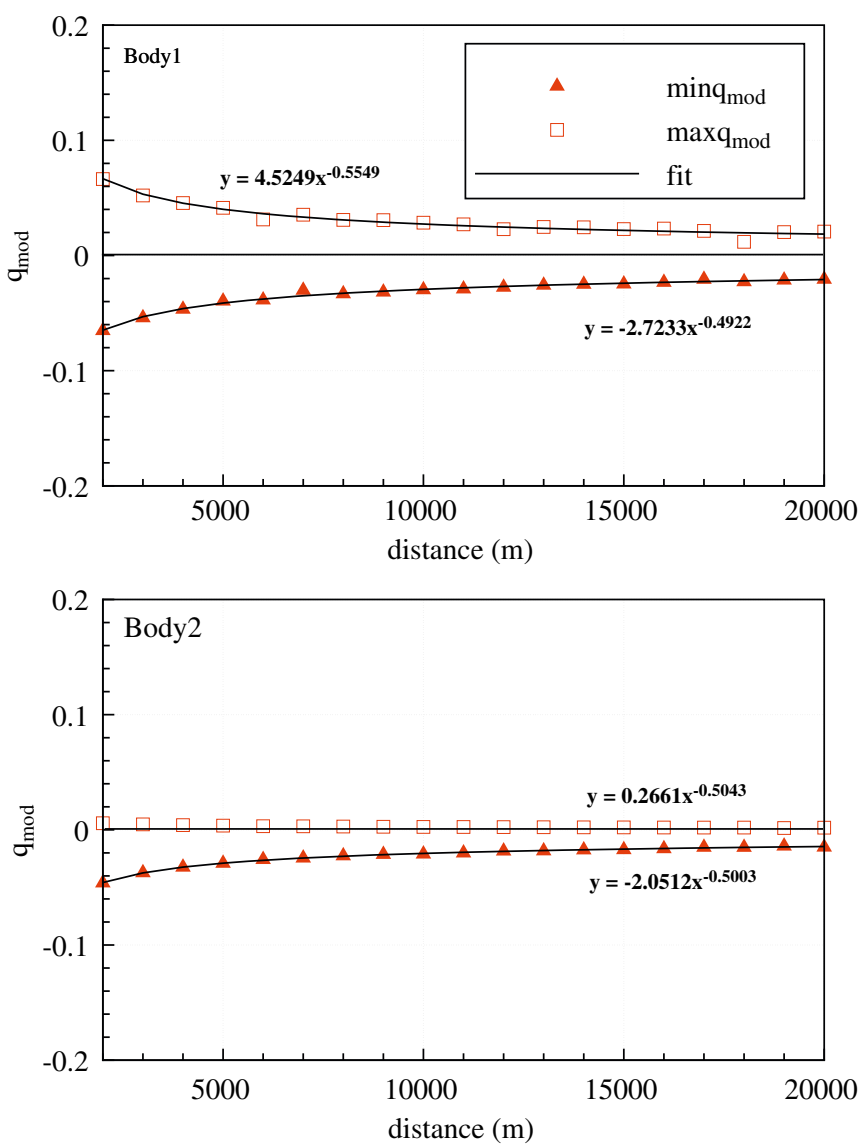

Fig. 9. Maximum and minimum $q_{\text {mod }}$ factors in array I at long separation distances. Top figure corresponds with body 1 and bottom figure with body 2 . The wave direction is $0^{\circ}$.

coefficient around -0.5 . This suggests that, at long distances, the wave interaction effect on the power absorption decreases with the square root of the distance. This is a bit surprising because, if the amplitude of the far field part of the wave decays with the square root of the distance, its energy decreases with the distance. Hence, one could have expected the wave interaction effect to decrease with the distance, not the square root of the distance. However, if one consider the way the wave energy is rebuilt behind the first body, one will find a dependency on the square root the distance (the proof of this last statement is given in appendix), which provide an explanation for such behaviour.

In Fig. 10, the $q_{\text {mod }}$ factor for both bodies 1 and 2 of array I have been plotted but in function of shorter distances from 100 to $2000 \mathrm{~m}$. One can see that the wave interactions effect is close to its asymptotic behaviour when the distance is longer than $1000 \mathrm{~m}$. For lower distances, the difference can be explained by nonnegligible near field effects.

More precisely, it appears in Fig. 10 that:

- The positive effect of wave interactions is much higher on body 1 than on body 2 .

- The negative effect of wave interactions is of the same order of magnitude for both bodies 1 and 2 .

- For body 1 , both positive and negative wave interactions effects remain significant (about $10 \%$ ) even when the two systems are separated by a distance higher than $1 \mathrm{~km}$. For body 2 , the positive effect is much smaller and decays rapidly with the distance. However, the maximum of negative effect appears to be still noticeable at large distances ( $5 \%$ at $2 \mathrm{~km}$ ).
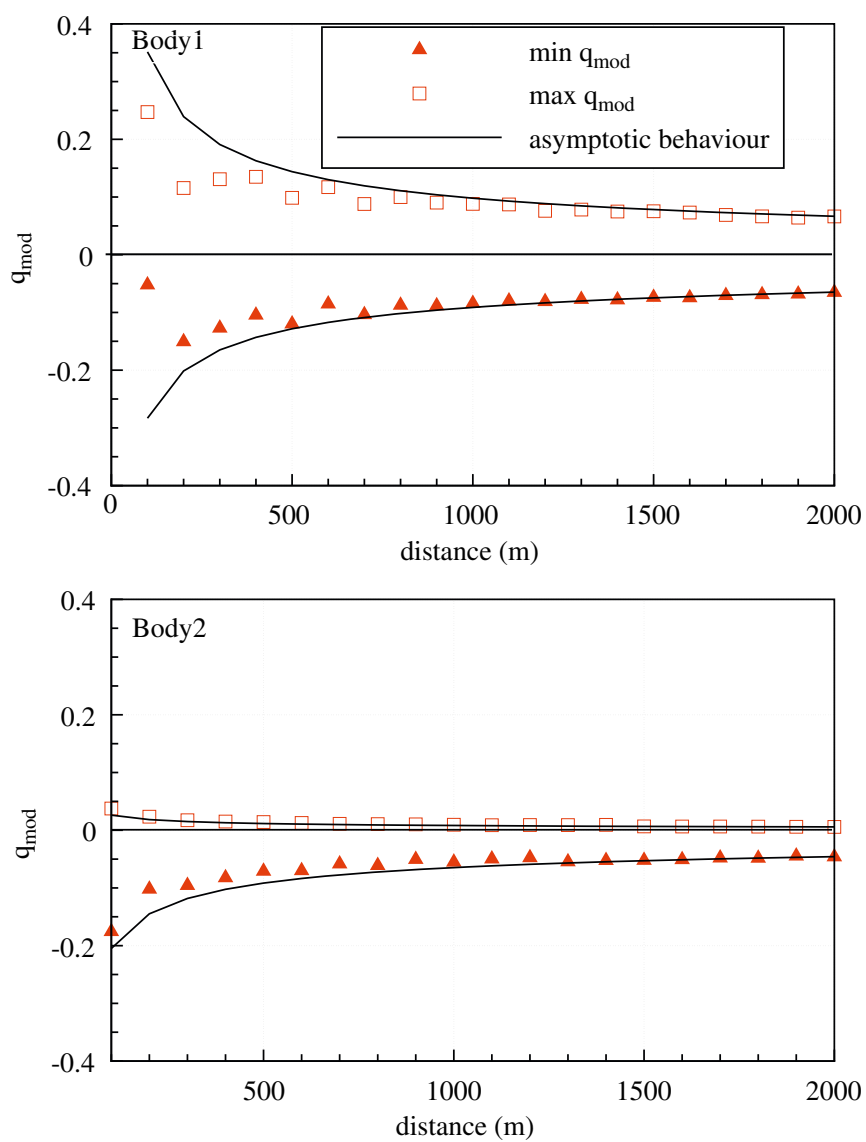

Fig. 10. Maximum and minimum $q_{\text {mod }}$ factors in array I at short separation distances. Top figure corresponds with body 1 and bottom figure with body 2 . The wave direction is 0 .

In Fig. 11, the case of array II is considered. One can see that, like in the previous case:

- The asymptotic behaviour of the wave interactions effect can be fitted using a polynomial law almost proportional with the square root of the distance.

- For short distances (shorter than $1800 \mathrm{~m}$ in this case), differences between the asymptotic behaviour and the computation become important. It shows that the effect of near wave field cannot be neglected anymore in the assessment of the wave interactions effect.

The positive effect is much higher on the first body than on the second one at short distances, and the negative one is of same order of magnitude. However, the trend is reversed for distances around $1800 \mathrm{~m}$, for which the positive effect becomes higher for body 2 than for body 1, which corresponds with the asymptotic behaviour. It shows that near field effects are responsible for this change in the behaviour of maximum of $q_{\text {mod }}$.

One could also notice that, in absolute value, the interaction effect is 3 times higher with these pontoon shapes in surge than in the case of the cylindrical shapes in heave. This leads to a still large wave interaction around $15 \%$ at a $2000 \mathrm{~m}$ distance.

\subsubsection{Influence of the wave direction}

Let us consider again array I but now with waves coming from a nonzero angle. In Fig. 12, the maximum and minimum of the $q_{\text {mod }}$ factor have been plotted in function of the distance between 

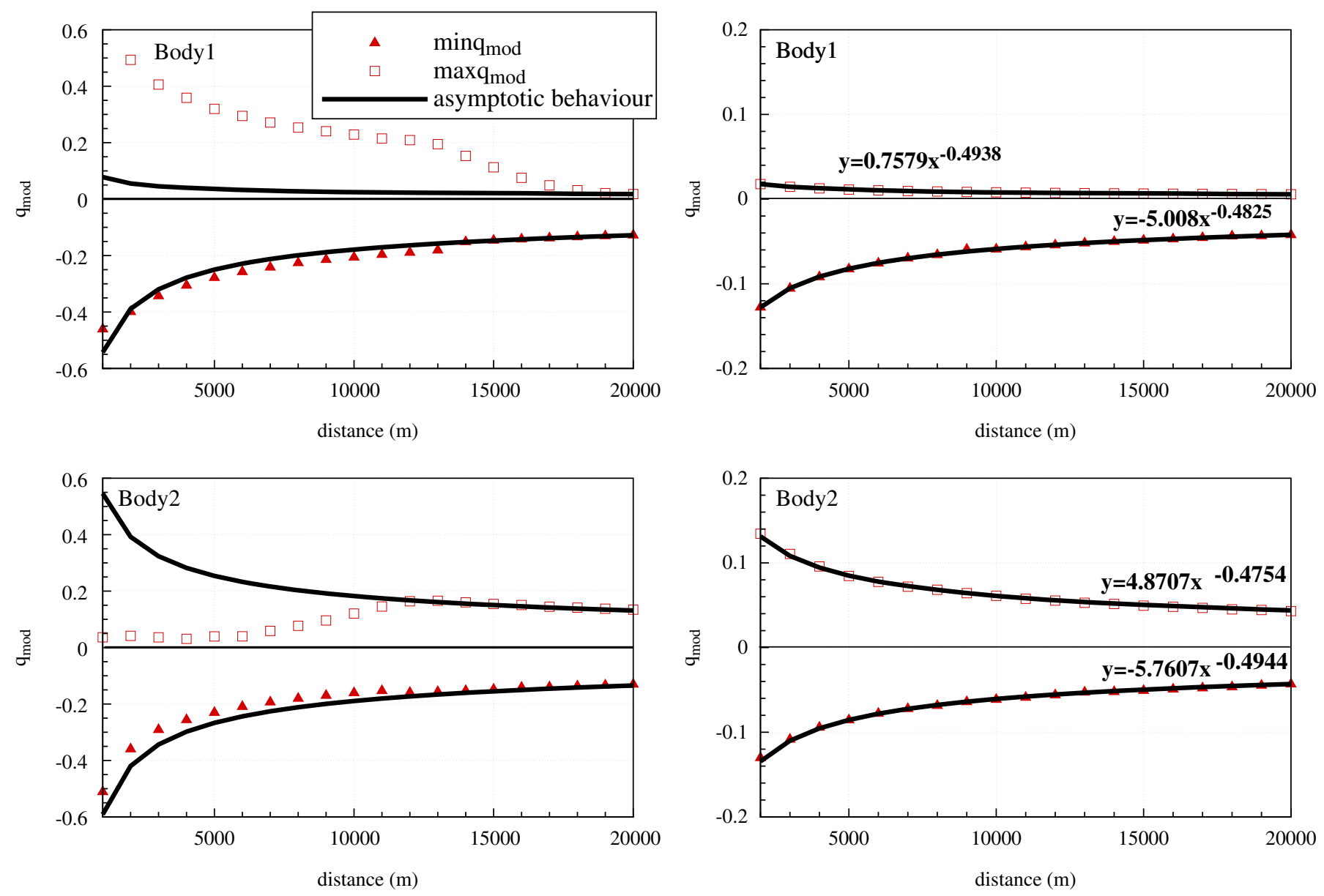

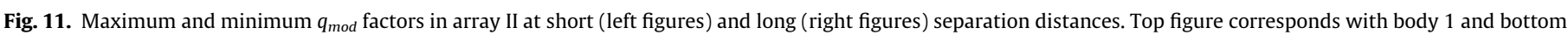
figure with body 2 . The wave direction is 0 .

the two systems for waves coming from the direction $0^{\circ}, 30^{\circ}, 60$ and $90^{\circ}$.

One can see that the wave direction affects the wave interaction effects, but not in the same way for the first and second body. On the first body, wave direction has an influence (oscillating with the distance) on the $q_{\text {mod }}$ factor, but only at short distances $(d<600 \mathrm{~m})$. Moreover, it does not modify its order of magnitude. On the second body, it is different: the maximum of the $q_{\text {mod }}$ factor is increased (up to a factor 6 in comparison with the $0^{\circ}$ wave direction) and both maximum and minimum of $q_{\text {mod }}$ shows a large influence (also oscillating with the distance) of the wave direction for all the range of distances considered. Hence, one can see that the wave interactions effect is stronger on body 2 when the two bodies are not aligned. However, it remains limited, but it is still around $10 \%$ at $2000 \mathrm{~m}$. One could notice that, for wave direction of $90^{\circ}$, the $q_{\text {mod }}$ factor is the same for both bodies 1 and 2 . This is due to obvious symmetry reasons.

In the case of array II, oscillations of the $q_{\text {mod }}$ factor can be observed for both bodies 1 and 2 in Fig. 13. However, the order of magnitude of the wave interaction effect is the same whatever the wave direction, except for the maximum of the $q_{\text {mod }}$ factor for body 1 at long distances, greatly increased. Here, it is assumed that it can be explained by near field effects, whose range would be increased for waves coming from a nonzero angle, but it has not been verified. Results with wave direction of $90^{\circ}$ have not been plotted on this figure because the $q_{\text {mod }}$ factor is not defined in this case (the absorbed power of an isolated system is 0 ).

\subsection{Irregular waves}

In regular waves, whatever the distance, the wave interaction effect can be negative $\left(q_{\text {mod }}<0\right)$ or positive $\left(q_{\text {mod }}>0\right)$ depending on the wave period. It can also remain quite large even at long distances $(10-15 \%$ at $2000 \mathrm{~m})$. In irregular waves and a fortiori when considering the whole annual energy production, one can expect the wave interaction effect to be smaller, because constructive and destructive interactions would compensate between each other.

In Fig. 14, the $q_{\text {mod }}$ factor of the mean annual wave power absorbed by bodies 1 and 2 of array I has been plotted in function of the distance separating the two bodies, for four wave directions.

For wave direction equal to $0^{\circ}$, wave interaction has a monotonous effect on each body. It is positive on the first body and negative on the second one. As it is expected, it decreases with the distance, but much faster for body 1 than for body 2 . Actually, for body 1, it appears to be almost unnoticeable for distances longer than $200 \mathrm{~m}$, whereas it still can be seen at a $2000 \mathrm{~m}$ distance for body 2 . However, one could notice that it is small, below $2 \%$ for distances longer than $200 \mathrm{~m}$.

For wave direction equal to $30^{\circ}$ and $60^{\circ}$, the wave interaction effect is more complex. It is not anymore monotonous, the positiveness or negativeness of the effect on each body being dependent on the distance. However, one can see that it decreases with the distance faster for body 1 than for body 2 and it 

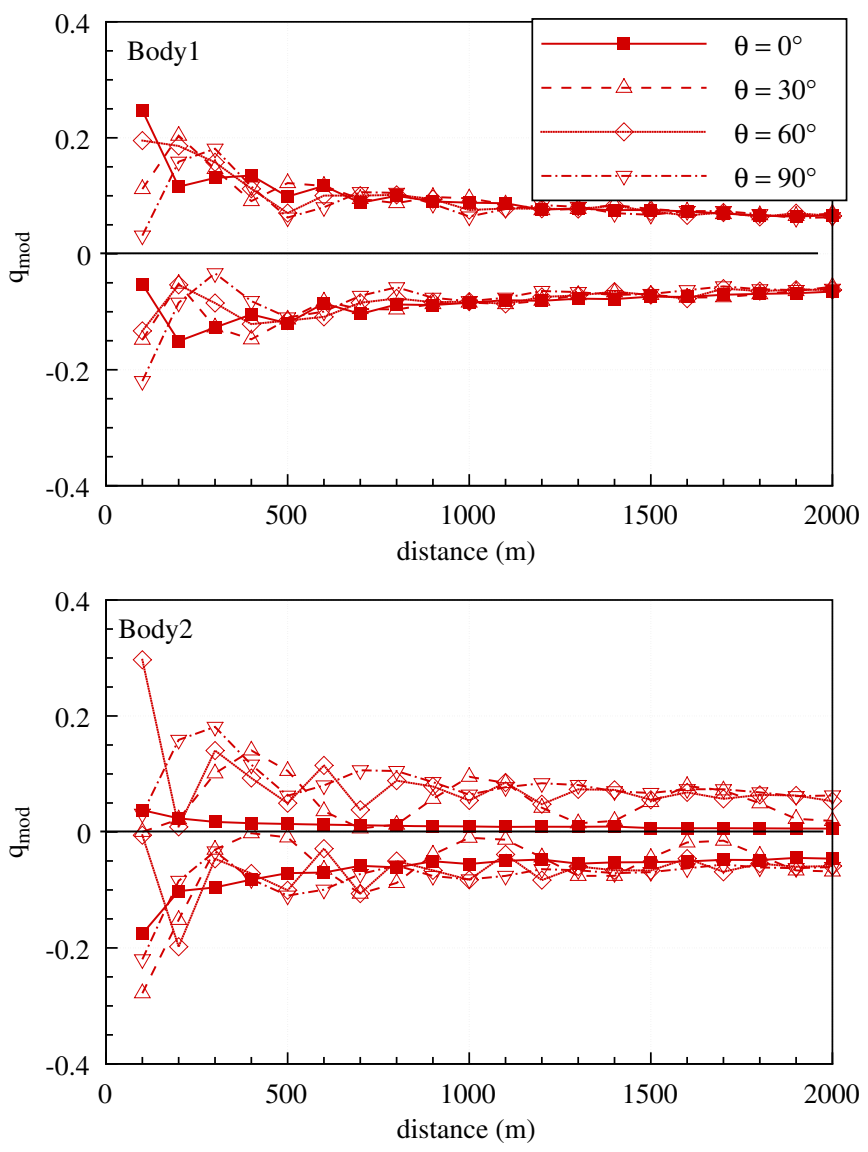

Fig. 12. Maximum and minimum $q_{\text {mod }}$ factors in array I in function of the distance for four different wave directions. Top figure corresponds with body 1 and bottom figure with body 2 .

becomes unnoticeable for distance greater than $400 \mathrm{~m}$ for body 1 . For body 2 , it has the behaviour of a sub critically damped oscillator-oscillating with respect to the separating distancewhose period seems to depend on the wave direction (longer for $30^{\circ}$ than for $60^{\circ}$ ). However, one could notice that it is not higher than $5 \%$ for distances greater than $400 \mathrm{~m}$.

For wave direction equal to $90^{\circ}$, the wave interaction effect is the same for both bodies 1 and 2 for symmetry reasons. It appears to be negative for distances smaller than $200 \mathrm{~m}$, positive for distances between 200 and $400 \mathrm{~m}$, and negligible for distances greater than $400 \mathrm{~m}$.

In an overall manner, one can see that the wave interaction effect on the absorbed power is rather small (smaller than $10 \%$ as soon as the distance exceeds $200 \mathrm{~m}$, even below $5 \%$ whatever the wave direction for distance greater than $500 \mathrm{~m}$ ). Regarding all the assumptions on which the numerical model is based, and all the uncertainties associated, it seems reasonable to ask ourselves if it is worth taking into account these wave interactions effect for distance greater than a few hundred meters.

In Fig. 15, the case of array II is considered. As in regular waves, results with wave direction of $90^{\circ}$ have not been plotted on this figure because of the $q_{\text {mod }}$ factor is not defined for this direction.

One can see that results are different from the case of array I. For wave direction equal to $0^{\circ}$, wave interaction is constructive for body 1 , negative for body 2 and decreases regularly for distances between 100 and $1200 \mathrm{~m}$. But the $q_{\text {mod }}$ factor curves of bodies 1 and 2 cross around $1200 \mathrm{~m}$, leading to the reversed effect for distances higher than $1200 \mathrm{~m}$. It increases for distance between 1200 and $1800 \mathrm{~m}$, then stabilises and finally decreases for longer
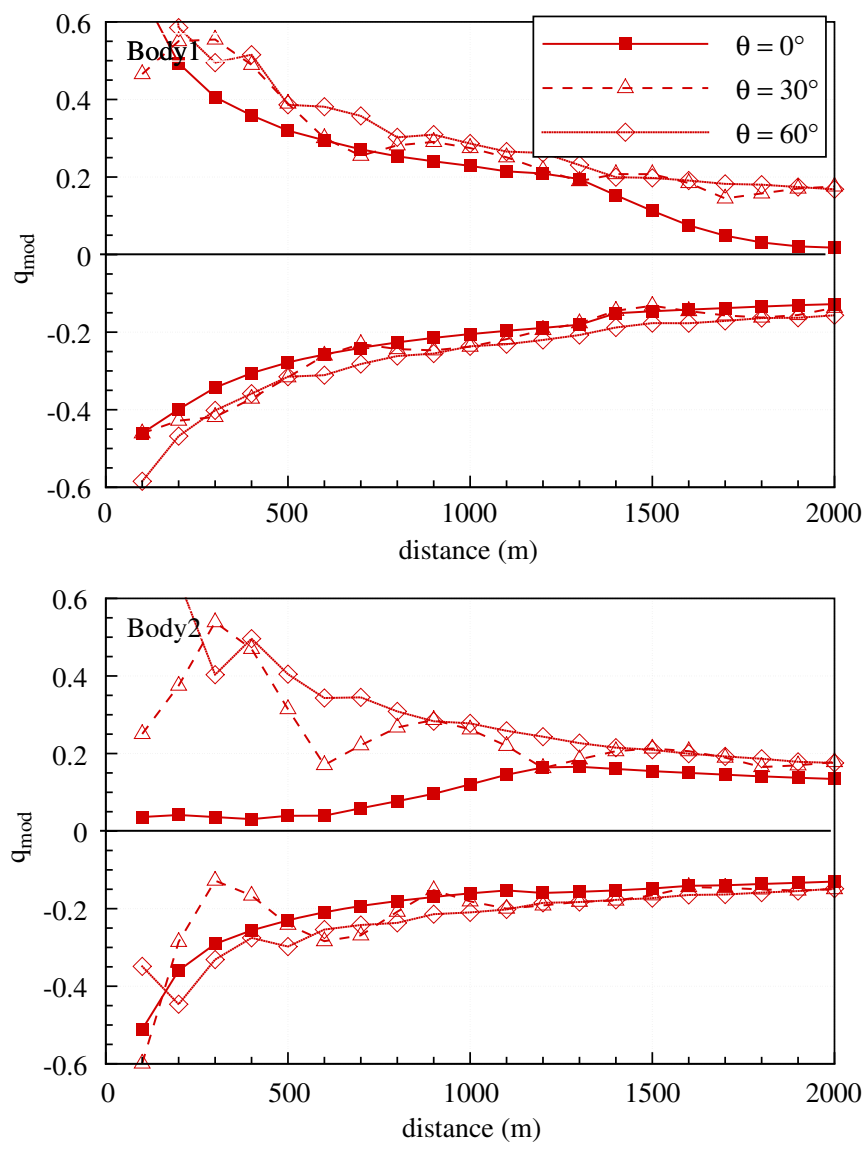

Fig. 13. Maximum and minimum $q_{\text {mod }}$ factors in array II in function of the distance for three different wave directions. Top figure corresponds with body 1 and bottom figure with body 2 .

distances, as it can be seen in Fig. 16. This is consistent with the results got in regular waves, for which it has been shown that near field effects were still noticeable for distances up to $1800 \mathrm{~m}$. It means that, in this case, near field effect leads to constructive interaction for body 1 and destructive interaction for body 2 , and far field effect leads to the reverse. At long distances, the last one will dominate, leading to a negative effect on body 1 and a positive one on body 2 (contrarily to the case of array I). However, overall, one could notice that the wave interaction is less than $10 \%$ as soon as the distance is greater than $400 \mathrm{~m}$.

For wave directions equal to $30^{\circ}$ and $60^{\circ}$, the wave interaction effect appears to be simpler. For body 1 , it is positive at all distances and smaller than $3 \%$ as soon as the distance is longer than $500 \mathrm{~m}$. For body 2, it is oscillating as in case of array I, but much more damped with the separating distance. It is also smaller than a few percents as soon as the distance is longer than $500 \mathrm{~m}$.

In Fig. 17, a comparison of the mean annual wave power absorbed by array I and array II has been plotted in dependence on the distance for the four wave directions. In case of array I, the device being omnidirectional, the total amount of wave power is not affected by the wave direction at long separating distances. One can see that the wave interaction effect appears to be negligible for distances greater than $500 \mathrm{~m}$.

For array II, the device being directional, the absorbed wave power is obviously affected by the wave direction. However, one can notice that, as for array I, the total amount of absorbed wave power is steady with the distance as soon as the bodies are separated by more than $500 \mathrm{~m}$. It shows that for greater separating distances, wave interactions effect can be neglected. 

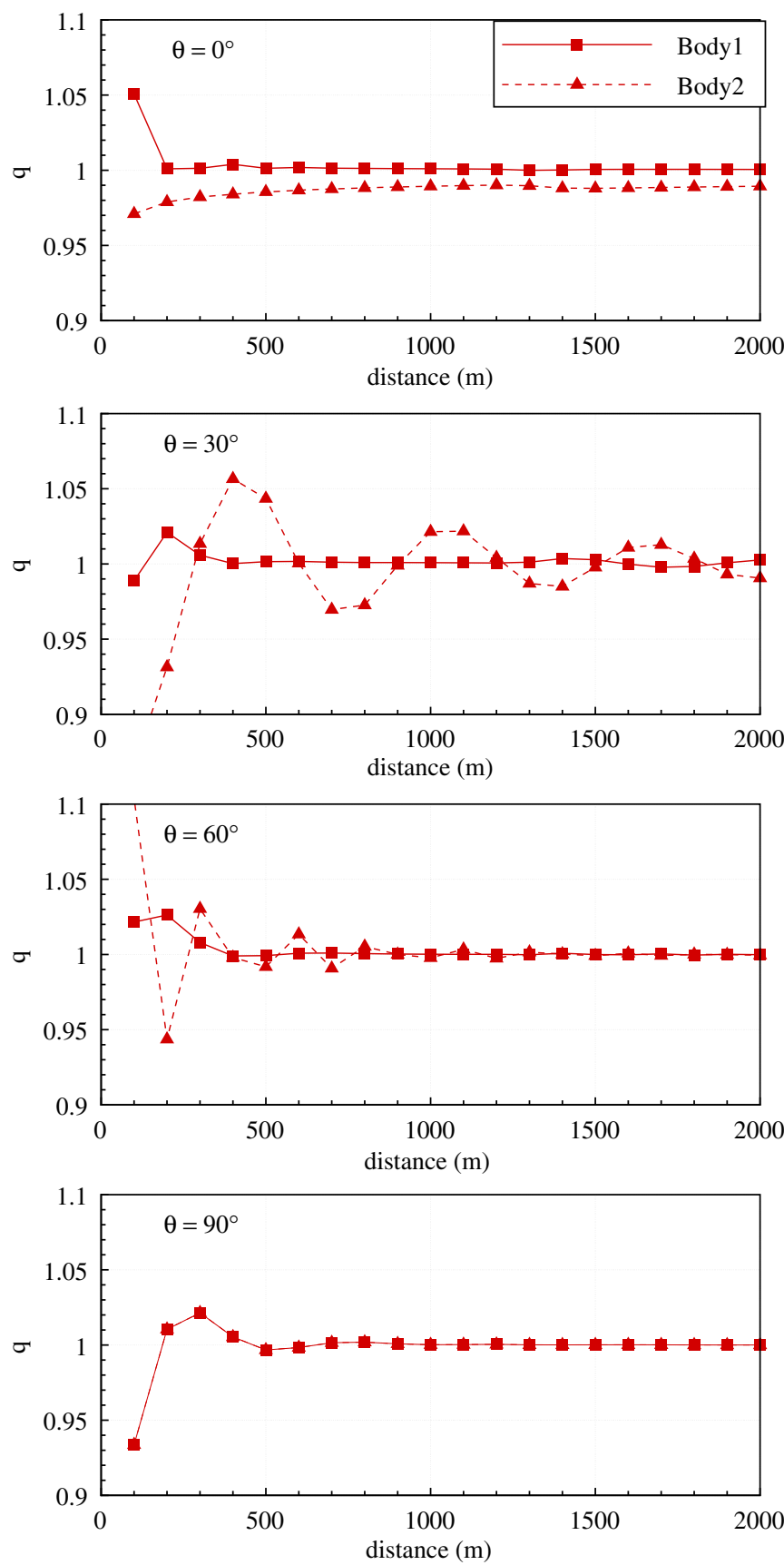

Fig. 14. $q_{\text {mod }}$ factor of the mean annual wave power absorbed by bodies 1 and 2 of array I in function of the distance for four different wave directions. For symmetry reasons, $q_{\text {mod }}$ of body 1 is equal to the one of body 2 when the wave direction is $90^{\circ}$.

\section{Conclusion}

In this paper, two arrays composed one of two heaving wave energy converters and the other of two surging WECs were considered. Parametric studies of the effects of the separating distance between the two systems and the wave direction on the wave interactions in the array and its wave energy absorption were conducted, both in regular and irregular waves.

In regular waves, a modified version of the $q$ factor is introduced and it is shown that it is a better indicator of the wave interaction effect on the wave power absorption than the usual $q$ factor. It is found that asymptotically, the absolute value of maxima and minima of this $q_{\text {mod }}$ factor decrease with the
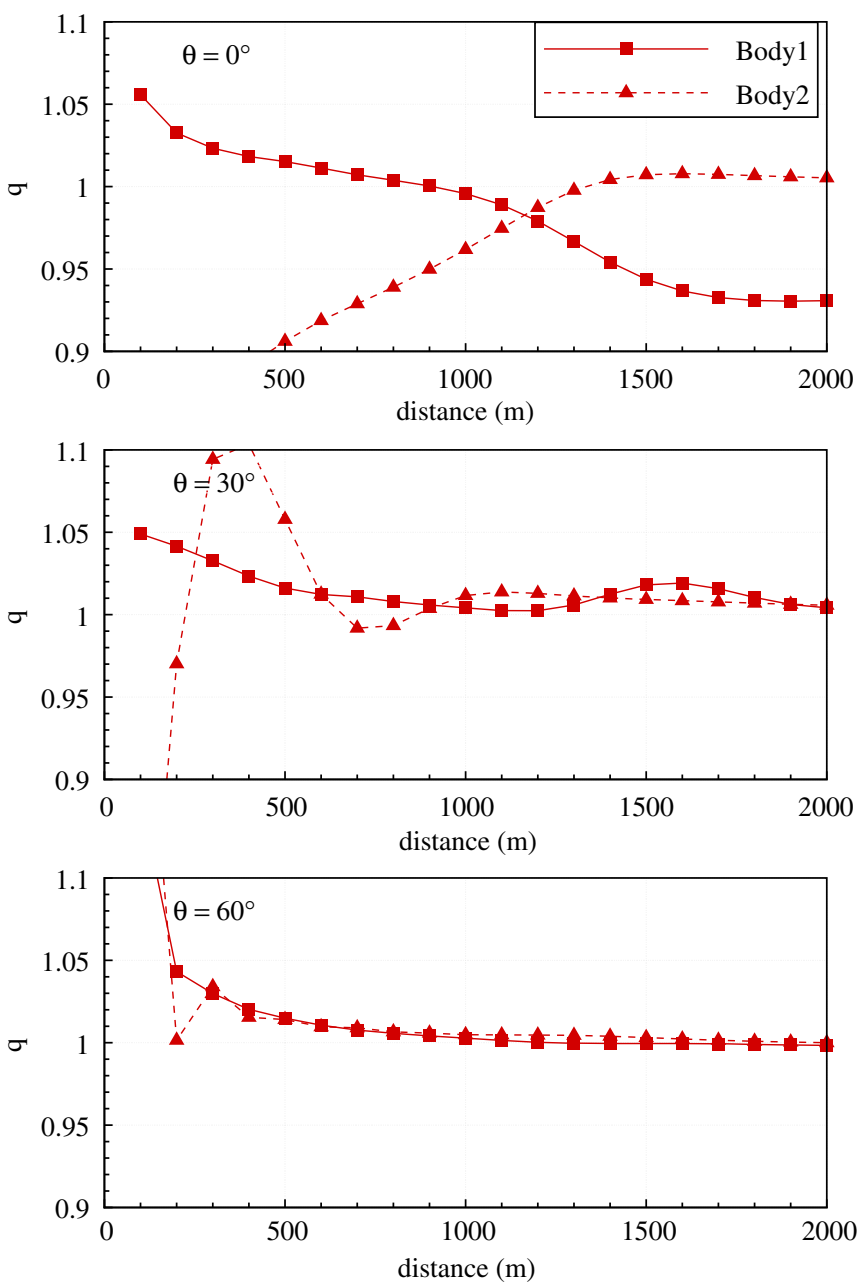

Fig. 15. $q_{\text {mod }}$ factor of the mean annual wave power absorbed by bodies 1 and 2 of array II in function of the distance for three different wave directions.

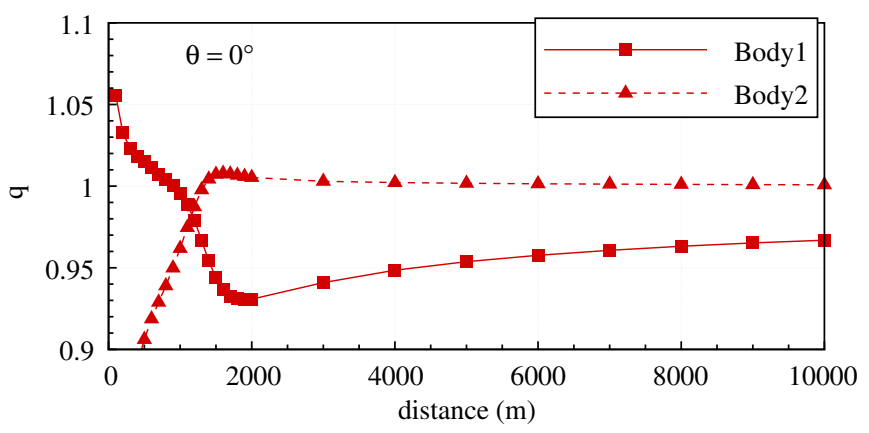

Fig. 16. $q_{\text {mod }}$ factor of the mean annual wave power absorbed by bodies 1 and 2 of array II at long distances. Wave direction is $0^{\circ}$.

square root of the distance, which is consistent with the way the wave energy is rebuilt behind the first body. This is a rather slow decay, which leads to a still noticeable impact on the absorbed power at long distance (maximum $10-15 \%$ at $2000 \mathrm{~m}$ ).

In irregular waves, it is shown that this impact on the mean annual absorbed power decreases faster with the distance. It is found to be, at maximum, less than $10 \%$ at $400 \mathrm{~m}$. Hence, reminding all the uncertainties associated with the assumptions on which is based the numerical model, it seems reasonable to neglect wave interaction effects for distance greater than $500 \mathrm{~m}$ 

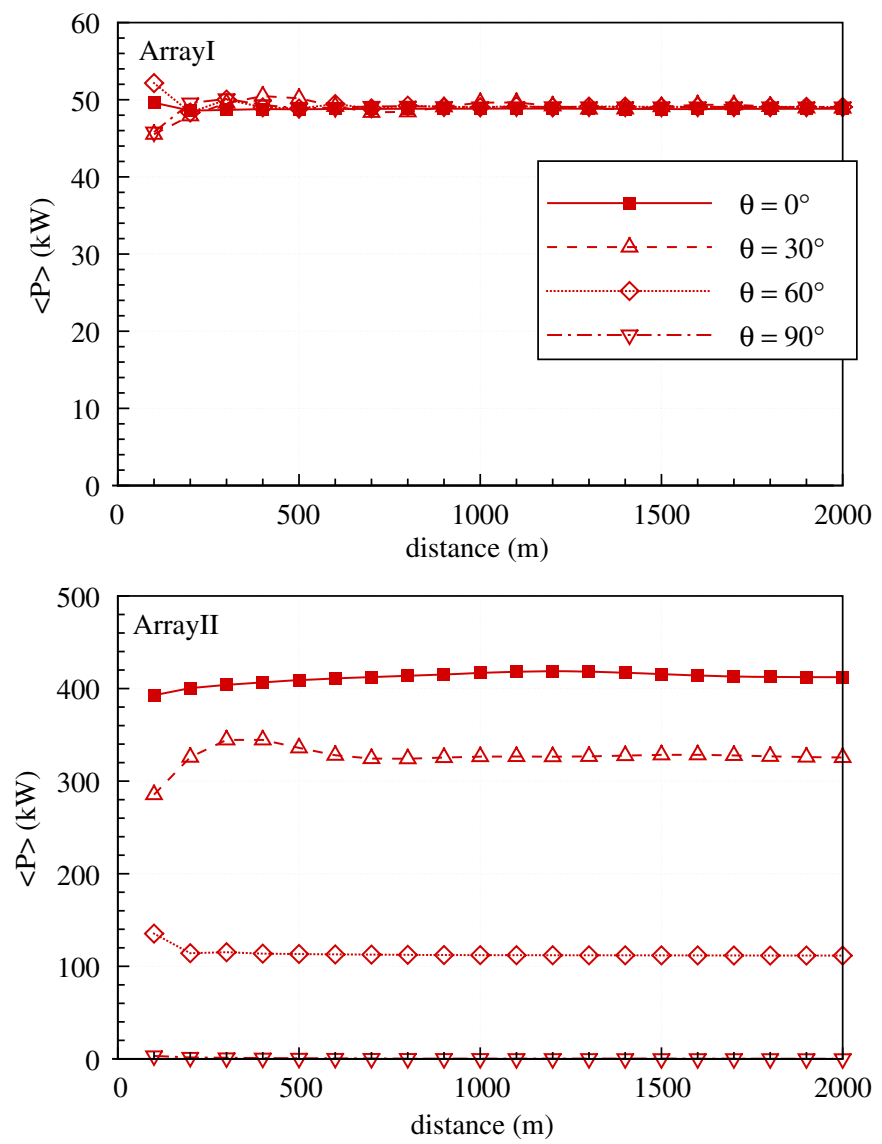

Fig. 17. Comparison of the mean annual power absorbed by array I (top) and array II (bottom) in function of the distance separating bodies 1 and 2 for four different wave directions.

when considering the mean annual absorbed power for the specific configuration studied here.

At short distances, shorter than $100 \mathrm{~m}$, it appears that it should not been neglected because the wave interaction can be significant for these distances.

At medium range, for distances between 100 and 500, it seems difficult to make any recommendation since the wave interaction can be low or high depending on the configuration, particularly when the wave direction is not aligned with the array.

Finally, these results were obtained with wave energy converters of typical dimensions of around $10 \mathrm{~m}$. It would be interesting to assess now how different dimensions of systems, particularly the width, would affect the distances defining these three ranges.

\section{Appendix A. Wave energy flux far behind a floating structure}

Let us consider the wave field around a single structure at a given point $M$. Let $(x, y, z)$ be the coordinates of $M$ in the reference frame of the structure, see Fig. 18.

According to the linear potential theory, the wave potential $\Phi$ can be decomposed into two:

$\Phi=\Phi_{I}+\Phi_{P}$

with:

- $\Phi_{I}$ being the incident undisturbed wave potential. In the case of a regular wave, one can write

$$
\Phi_{I}(x, y, z, t)=A \Im\left(\left(\frac{i g}{\omega}\right) e^{k z+i(k x-\omega t)}\right)
$$

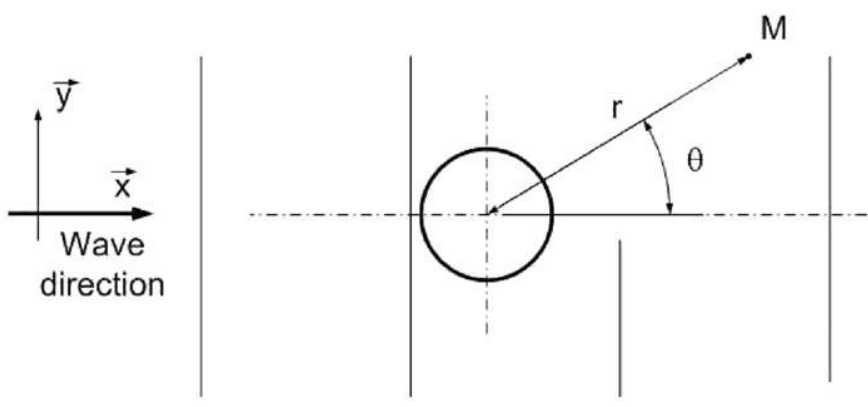

Fig. 18. Notations used for the calculation of wave energy flux far behind a floating system.

in which $A$ is the wave amplitude, $k$ is the wave number and $\omega$ is the circular wave frequency.

- $\Phi_{P}$ being the perturbation potential corresponding with diffracted and radiated waves.

Assuming to be sufficiently far from the source of the perturbation, one can write $\Phi_{P}$ as (Mei et al., 2005)

$\left.\Phi_{P}=A \Im \sqrt{\frac{k}{2 \pi r}} e^{k z+i(k r-\omega t-\pi / 4)} H(\theta)\right)$

in which $(r, \theta, z)$ are the circular coordinates of $M$-i.e. $r=\sqrt{x^{2}+y^{2}}$ and $\theta$ such as $x=r \cos \theta$ and $y=r \sin \theta$ - and $H(\theta)$ is the so-called complex Kochin function. This Kochin function, which depends only on the direction of propagation $\theta$, fully characterises the far field component of the diffraction-radiation potential.

From Eqs. (A.2) and (A.3), let us derive the pressure $p$ and the flow velocity $\vec{V}$ :

$\frac{p}{\rho g A}=\mathfrak{J}\left(\left[e^{i k x}-\frac{i \omega}{g} \sqrt{\frac{k}{2 \pi r}} e^{i(k r-\pi / 4)} H(\theta)\right] e^{k z-i \omega t}\right)$

$\left.\frac{\vec{V}}{A}=\mathfrak{J}\left(\left[\begin{array}{c}-\omega \cos \theta \\ \omega \sin \theta \\ i \omega\end{array}\right) e^{i k x}+\left(\begin{array}{c}-\frac{1}{2 r}+i k \\ H^{\prime}(\theta) / H(\theta) \\ k\end{array}\right) \sqrt{\frac{k}{2 \pi r}} e^{i(k r-\pi / 4)} H(\theta)\right] e^{k z-i \omega t}\right)$

These two last equations are valid everywhere in the fluid domain, provided the distance $r$ to be sufficient. Let us focus now on the variation of the wave along the $x$-axis, behind the system, i.e. $\theta=0$ and $r=x$. Eqs. (A.4) and (A.5) become

$\frac{p}{\rho g A}=\mathfrak{J}\left(\left[1-\frac{i \omega}{g} \sqrt{\frac{k}{2 \pi x}} e^{-i \pi / 4} H(0)\right] e^{k(z+i x)-i \omega t}\right)$

$\frac{\vec{V}}{A}=\mathfrak{J}\left(\left[\left(\begin{array}{c}-\omega \\ 0 \\ i \omega\end{array}\right)+\left(\begin{array}{c}-\frac{1}{2 x}+i k \\ H^{\prime}(0) / H(0) \\ k\end{array}\right) \sqrt{\frac{k}{2 \pi x}} e^{-i \pi / 4} H(0)\right] e^{k(z+i x)-i \omega t}\right)$

The mean flux of wave energy propagating along the $x$-axis per meter of wave front is given by

$W=-\int_{-\infty}^{0} \int_{0}^{T} p \vec{V} \cdot \vec{x} d t d z$

Using Eqs. (A.6) and (A.7), one can show

$\frac{W}{\rho g A^{2}}=-\frac{1}{4 k} \Re\left(\left(-\omega+\left(-\frac{1}{2 x}+i k\right) \sqrt{\frac{k}{2 \pi x}} e^{-i \pi / 4} H(0)\right)\left(1+\frac{i \omega}{g} \sqrt{\frac{k}{2 \pi x}} e^{i \pi / 4} H^{*}(0)\right)\right)$

in which $H^{*}(0)$ is the complex conjugate of $H(0)$. Finally, neglecting the terms of order higher than $\sqrt{1 / x}$, one can get 
for $W$ :

$W \simeq \frac{\rho g^{2} A^{2}}{4 \omega}\left(1+\frac{2 \omega}{g} \sqrt{\frac{k}{2 \pi x}} \Im\left(e^{-i \pi / 4} H(0)\right)\right)$

in which one recognises the addition of the wave energy flux of the incident wave $\rho g^{2} A^{2} / 4 \omega$ plus a correction which decays as the square root of the distance. Hence, one can see that the rate of recovery of the wave energy flux behind a wave energy system is proportional to the inverse of the square root of the distance.

\section{References}

Budal, K., 1977. Theory of absorption of wave power by a system of interacting bodies. Journal of Ship Research 21, 248-253.

Child B., Venugopal V., 2007. Interaction of waves with an array of floating wave energy devices. In: Proceedings of the 7th European Wave and Tidal Energy Conference, Porto, Portugal.

Cruz J., Sykes R., Siddorn P., Eatock Taylor, R., 2009. Wave farm design: preliminary studies on the influences of wave climate, array layout and farm control. In: Proceedings of the 8th European Wave and Tidal Energy Conference, Uppsala, Sweden, pp. 736-745.

Delhommeau G., 1993. Seakeeping codes AQUADYN and AQUAPLUS. In: Proceedings of the 19th WEGEMT SCHOOL on Numerical Simulation of Hydrodynamics: Ships and Offshore Structures, Nantes, France.
Evans D.V., 1979. Some theoretical aspects of three-dimensional wave-energy absorbers. In: Proceedings of the 1st Symposium on Wave Energy Utilisation, Gothenburg, Sweden.

Falcão de, A.F.O., 2002. Wave-power absorption by a periodic linear array of oscillating water columns. Ocean Engineering 29, 1163-1186.

Falnes, J., 1980. Radiation impedance matrix and optimum power absorption for interacting oscillators in surface waves. Applied Ocean Research 2, 75-80.

Falnes, J., 2002. Ocean Waves and Oscillating Systems, Linear Interactions Including Wave-energy Extraction, first ed. Cambridge University Press, Cambridge.

Justino, P.A.P., Clément A.H., 2003. Hydrodynamic performance for small arrays of submerged spheres. In: Proceedings of the 5th European Wave Energy Conference, Cork, Ireland.

Mavrakos, S.A., Mclver, P., 1997. Comparison of methods for computing hydrodynamic characteristics of arrays of wave power devices. Applied Ocean Research 19, 283-291.

McIver, P., Evans, D.V., 1984. Approximation of wave forces on cylinder arrays. Applied Ocean Research 6, 101-107.

Mei, C.C., Stiassnie, M., Yue, D.K.-P., 2005. Theory and Applications of Ocean Surface Waves. Part 1: Linear Aspects, first ed. World Scientific, Singapore.

Molin, B., 2002. Hydrodynamique des structures offshore. Guides pratiques sur les ouvrages en mer. TECHNIP editor.

Simon, M.J., 1982. Multiple scattering in arrays of axisymmetric wave-energy devices. Part 1. A matrix method using a plane-wave approximation. Journal of Fluid Mechanics 120, 1-25.

Weller S., Stallard, T., Stansby, P.K., 2009. Experimental measurements of irregular wave interaction factors in closely spaced arrays. In: Proceedings of the 8th European Wave and Tidal Energy Conference, Uppsala, Sweden.

Thomas, G.P., Evans, D.V., 1981. Arrays of three-dimensional wave-energy absorbers. Journal of Fluid Mechanics 108, 67-88. 